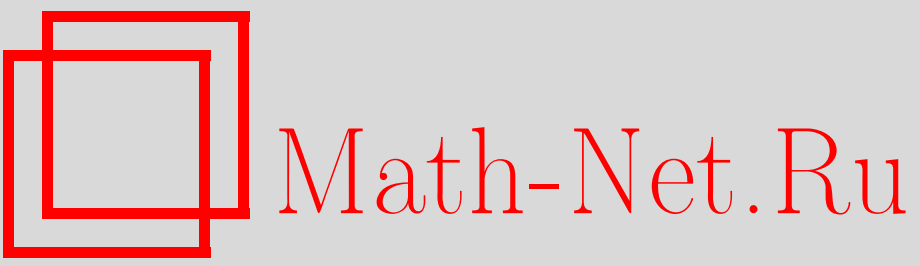

Л. О’Раферти, Д. М. Павловски, В. В. Сридар, К вопросу о дуальности квантовой теории поля Лиувилля, ТМФ, 2000, том 123, номер 2, 299-307

DOI: https://doi.org/10.4213/tmf604

Использование Общероссийского математического портала Math-Net.Ru подразумевает, что вы прочитали и согласны с пользовательским соглашением

http://www.mathnet.ru/rus/agreement

Параметры загрузки:

IP: 54.172 .240 .79

26 апреля 2023 г., 14:17:16 
ТЕОРЕТИЧЕСКАЯ

И МАТЕМАТИЧЕСКАЯ

ФИЗИКА

Том 123, № 2

май, 2000

(C) 2000 г. Л. О’ Раферти*, Дж.М. Павловски*, В.В. Сридар*

\title{
К ВОПРОСУ О ДУАЛЬНОСТИ КВАНТОВОЙ ТЕОРИИ ПОЛЯ ЛИУВИЛЛЯ
}

\begin{abstract}
Обнаружено, что центр алгебры Вирасоро и трехточечные функции квантовой теории поля Лиувилля с потенциалом $\exp (2 b \phi(x))$ и внешними примарными полями $\exp (\alpha \phi(x))$ инвариантны по отношению к преобразованиям дуальности $\hbar \alpha \rightarrow q-\alpha$, где $q=b^{-1}+b$. Шаги, приводящие к этому результату (использующие алгебру Вирасоро и трехточечные функции), описаны в терминах формализма интеграла по путям. Дуальность возникает в связи с тем, что в квантовом случае одному значению конформного веса $\Delta_{\alpha}$ отвечают два в общем случае различных значения $\alpha$. В результате квантовый потенциал Лиувилля может содержать две экспоненты (с соответствующими параметрами). Показано, что в теории с двумя экспонентами дуальность возникает естественным образом и важная экстраполяция, о возможности которой предполагалось ранее, может быть доказана.
\end{abstract}

\section{1. ВВЕДЕНИЕ}

Интерес к двумерной теории Лиувилля [1] обусловлен тем, что это нетривиальная конформно-инвариантная теория поля, включающая единственное (скалярное) поле. Кроме того, эта теория часто упоминалась в последнее время в различных контекстах, особенно в связи с теорией струн $[2,3]$. Однако квантовая формулировка этой теории сопряжена с некоторыми проблемами. Например, в противоположность четырехточечной функции Весса-Зумино-Виттена (ВЗВ) четырехточечная функция Лиувилля не может быть выражена в терминах элементарных аналитических функций (аналог уравнения Книжника-Замолодчикова имеет второй порядок). Даже для трехточечной функции, пространственно-временная зависимость которой фиксируется конформной инвариантностью, коэффициенты рассчитать трудно. С другой стороны, то, что известно о трехточечных функциях, представляется весьма интересным, и для этой теории характерна дуальность, которой нет на классическом уровне $[4,5]$. В настояшей статье мы хотели бы сделать обзор квантовой теории Лиувилля с точки зрения интеграла по путям и обсудить соответствующую дуальность. Сперва будет дано описание этой теории, а затем предложен подход, в рамках которого она может быть включена в функциональный интеграл посредством использования потенциала с двумя экспонентами.

*Dublin Institute for Advanced Studies. Ireland. E-mail: physics@stp.dias.ie 


\section{2. ИНТЕГРАЛ ПО ПУТЯМ В ТЕОРИИ ЛИУВИЛЛЯ}

Классическая теория Лиувилля определяется действием

$$
S(\phi)=\int d^{2} x\left[\frac{1}{2}(\partial \phi)^{2}+\mu e^{2 b \phi}\right]
$$

где $\mu$ и $b$-константы, $\phi(x)$ - скалярное поле, и в двух измерениях это действие конформно-инвариантно. Если эту теорию применить к искривленному пространству посредством введения действия обобшенного вида

$$
S(\phi)=\int d^{2} x\left[\frac{1}{2} \sqrt{g} g^{\mu \nu}\left(\partial_{\mu} \phi\right)\left(\partial_{\nu} \phi\right)+\mu \sqrt{g} e^{2 b \phi}+q \sqrt{g} R \phi\right],
$$

где $R$ - кривизна Римана, а $q$ - некоторая постоянная, то конформная инвариантность переходит в инвариантность Вейля, т.е. инвариантность по отношению к преобразованию $g_{\mu \nu}(x) \rightarrow \lambda(x) g_{\mu \nu}(x)$ для любого гармонического масштабного фактора $\lambda(x)$, если только константа $q$ есть

$$
q=b^{-1} \quad \text { и } \quad q=b^{-1}+\hbar b
$$

в классической и квантовой теориях, соответственно. Квантовая дуальность проявляется уже на этой стадии в том, что $q$ инвариантна относительно преобразования $b \leftrightarrow(\hbar b)^{-1}$.

Варьируя $g^{\mu \nu}$ в $(2.2)$ и затем переходя к пределу плоского пространства, можно видеть, что тензор энергии-импульса есть

$$
T_{\mu \nu}=\left(\partial_{\mu} \phi\right)\left(\partial_{\nu} \phi\right)-\frac{1}{2} \eta_{\mu \nu}(\partial \phi)^{2}+q\left(\eta_{\mu \nu} \partial^{2} \phi-\partial_{\mu} \partial_{\nu} \phi\right),
$$

где $\eta$ - метрика Минковского. Этот тензор энергии-импульса обладает нулевым следом на массовой поверхности и приводит к операторам Вирасоро [6-8] в форме

$$
T_{++}=\left(\partial_{+} \phi\right)^{2}-q \partial_{+}^{2} \phi \quad \text { и } \quad T_{--}=\left(\partial_{-} \phi\right)^{2}-q \partial_{-}^{2} \phi .
$$

Для этих операторов Вирасоро центры имеют вид

$$
c=\hbar+k q^{2},
$$

где $k$ - численная константа, а поля $e^{\alpha \phi}$ суть примарные поля конформных весов $\left(\Delta_{\alpha}, \Delta_{\alpha}\right)$, где

$$
\Delta_{\alpha}=\alpha(q-\hbar \alpha),
$$

причем $\alpha q$ появляется из поправочного члена, а $\alpha^{2}$ - в связи с нормальным упорядочением стандартного тензора энергии-импульса. Другое проявление дуальности на этой стадии состоит в том, что конформные веса $\hbar \Delta_{\alpha}$ инвариантны по отношению к преобразованию $\hbar \alpha \leftrightarrow(q-\hbar \alpha)$. Эта вторая форма дуальности является более обшей, чем первая, которая, по существу, есть ее частный случай с $\Delta_{\alpha}=1$. Немедленное следствие дуальности - наличие в квантовых конформных теориях поля двух примарных полей вида $e^{2 \alpha \phi}$ для каждого конформного веса $\Delta_{\alpha}$. Другими словами, сушествуют два веса

$$
\alpha_{ \pm}=\frac{q \pm \sqrt{q^{2}-4 \hbar \Delta_{\alpha}}}{2 \hbar}
$$


для любого $\Delta_{\alpha}$. Заметим, что в пределе $\hbar \rightarrow 0$ имеем $\alpha_{+} \rightarrow \infty$ и $\alpha_{-} \rightarrow \alpha$, так что только один корень остается конечным.

Далее мы будем полагать, что $\hbar=1$ и что двумерное пространство является сферой, а также использовать конформные координаты, т.е. координаты, для которых

$$
g_{\mu \nu}(x)=e^{\sigma(x)} \eta_{\mu \nu}
$$

В этих координатах действие редуцируется к виду

$$
S(\phi)=\int d^{2} x\left[\frac{1}{2} \eta^{\mu \nu}\left(\partial_{\mu} \phi\right)\left(\partial_{\nu} \phi\right)+\mu e^{2(b \phi+\sigma)}+q e^{2 \sigma} R \phi\right] .
$$

\section{N-ТОЧЕЧНЫЕ ФУНКЦИИ}

В терминах интеграла по путям мы можем записать $N$-точечную функцию в виде

$$
\left\langle\prod_{a} e^{2 \alpha_{a} \tilde{\phi}\left(x_{a}\right)}\right\rangle=\int d \tilde{\phi}(x) e^{i S(\tilde{\phi}(x))} \prod_{a} e^{2 \alpha_{a} \tilde{\phi}\left(x_{a}\right)} .
$$

Экспоненциальный потенциал имеет следуюшее свойство: если выделить нулевую моду $\phi_{0}$ :

$$
\tilde{\phi}(x)=\phi_{0}+\phi(x), \quad \int d^{2} x \phi(x)=0
$$

то интегрирование в (3.1) по нулевой моде может быть отделено от остальной части [9]:

$$
\left\langle\prod_{a} e^{2 \alpha_{a} \tilde{\phi}\left(x_{a}\right)}\right\rangle=\int d \phi e^{i S(\phi)} \prod_{a} e^{2 \alpha_{a} \phi\left(x_{a}\right)}\left(\int d \phi_{0} \exp \left\{-2 \xi \phi_{0}+e^{2 b \phi_{0}} V_{b}(\phi)\right\}\right)
$$

где

$$
\xi=q-\sum_{a} \alpha_{a}, \quad V_{b}(\phi)=\mu e^{2 b \phi}
$$

при этом был использован тот факт, что для сферы выполняется равенство $\int d^{2} x \sqrt{g} R=\chi=2$, где $\chi$ - число Эйлера. Далее, с точностью до некоторых множителей интеграл по нулевой моде в (3.3) есть просто интеграл для Г-функции, так что его легко упростить:

$$
\prod_{a}\left\langle e^{2 \alpha_{a} \tilde{\phi}\left(x_{a}\right)}\right\rangle=\Gamma\left(-\frac{\xi}{b}\right) \int d \phi e^{i S(\phi)} \prod_{a} e^{2 \alpha_{a} \phi\left(x_{a}\right)}\left(V_{b}(\phi)\right)^{\frac{\xi}{b}} .
$$

Здесь мы сталкиваемся с серьезными трудностями теории Лиувилля, связанными с тем, что интеграл по путям может быть вычислен, только когда $\xi / b$ принимает положительные целые значения, а именно в этих точках коэффициент $\Gamma(-\xi / b)$ имеет полюсы. Поэтому на практике мы можем лишь рассчитать вычеты в полюсах и затем попытаться экстраполировать их на другие точки. Следует принять во внимание, что вычеты

$$
R_{N}\left(x_{a}, \alpha_{a}, m\right)=\frac{(-1)^{m}}{m !} \int d \phi e^{i S(\phi)} \prod_{a} e^{2 \alpha_{a} \phi\left(x_{a}\right)}\left(V_{b}(\phi)\right)^{m}
$$


оказываются интегралами свободного поля, которые сушествуют в литературе [10]. Более точно, если выделить стандартный фактор Полякова $\exp \left(1+k q^{2}\right) \int R \Delta^{-2} R$, включающий конформную аномалию, выполнить обычную перенормировку, выделяя сингулярности функции Грина, и перейти к пределу плоского пространства, то можно получить вычеты

$$
R_{N}\left(x_{a}, \alpha_{a}, m\right)=\frac{(-\mu)^{m}}{m !} \prod_{a \neq b}\left|x_{a b}\right|^{-2 \alpha_{a} \alpha_{b}} \prod_{i \neq j}^{i=m} \int d^{2} x_{i}\left|x_{i j}\right|^{-2 b^{2}}\left|x_{i a}\right|^{-4 b \alpha_{a}}
$$

где $x_{a b} \equiv x_{a}-x_{b}$. Из конформной инвариантности следует, что ядро интеграла во втором сомножителе $S L(2, C)$-инвариантно. Поскольку группа $S L(2, C)$ определяется тремя параметрами, статистическая сумма и одноточечная функция не существуют, в то время как двухточечная функция имеет фактор $\delta\left(\Delta_{1}-\Delta_{2}\right)$, где величины $\Delta$ суть конформные веса двух внешних полей. Наиболее интересен случай $N=3$, когда вычет принимает форму

$$
R_{3}\left(x_{a}, \alpha_{a}, m\right)=C_{3}\left(\alpha_{a}, m\right) \prod_{a \neq b}^{3}\left|x_{a b}\right|^{-2 \Delta_{a b}},
$$

где $C_{3}$ - численньй коэффициент, а

$$
\Delta_{a b}=\Delta_{a}+\Delta_{b}-\Delta_{c}, \quad \Delta_{a}=\alpha_{a}\left(q-\alpha_{a}\right)
$$

При $N>3$ вычеты имеют форму

$$
R_{N}\left(x_{a}, \alpha_{a}, m\right)=\left|\frac{x_{23}}{x_{12} x_{31}}\right|^{2 \xi(\xi+q)} \prod_{a \geqslant 2}\left|x_{1 a}\right|^{4 \xi \alpha_{a}} K\left(\alpha_{a}, r_{c}\right)
$$

где $r_{c}(x)$ суть конформно-инвариантные отношения вида $r_{c}=x_{c 2} x_{31} / x_{c 1} x_{23}$ и

$$
K\left(\alpha_{a}, r_{c}\right)=\prod_{c \geqslant 4} \int d^{2} x_{i}\left|x_{i j}\right|^{-2 b^{2}}\left|x_{i}\right|^{-4 b \alpha_{2}}\left|x_{i}-1\right|^{-4 b \alpha_{3}}\left|x_{i}-r_{c}\right|^{-4 b \alpha_{c}} .
$$

\section{4. КОЭФФИЦИЕНТЫ ДЛЯ ТРЕХТОЧЕЧНЫХ ФУНКЦИЙ}

Значения коэффициента $C_{3}\left(\alpha_{a},-\xi / b\right)$ трехточечной функции в полюсах $\xi=-m b$ Г-функции могут быть вычеслены из интеграла свободного поля

$$
C_{3}\left(\alpha_{a}, m\right)=\frac{(-\mu)^{m}}{m !} \prod_{i \neq j}^{m} \int d^{2} x_{i}\left|x_{i j}\right|^{-2 b^{2}}\left|x_{i a}\right|^{-4 b \alpha_{a}}
$$

которьй может быть взят [10]:

$$
\begin{aligned}
C_{3}\left(\alpha_{a}, m\right)= & \left(\mu_{r}\right)^{m b} \frac{k^{\prime}(0)}{k^{\prime}(-m b)} \times \\
& \times\left[\frac{k\left(2 \alpha_{1}\right) k\left(2 \alpha_{2}\right) k\left(2 \alpha_{3}\right)}{k\left(\alpha_{1}+\alpha_{2}-\alpha_{3}\right) k\left(\alpha_{2}+\alpha_{3}-\alpha_{1}\right) k\left(\alpha_{3}+\alpha_{1}-\alpha_{2}\right)}\right],
\end{aligned}
$$


где $\mu_{r}=\left[-\mu \gamma\left(b^{2}\right)\right]^{1 / b}$, а функция $k(\alpha)$, которая неявно зависит от $b$, определяется следуюшими замечательными свойствами:

$$
\begin{aligned}
k(\alpha) & =k(q-\alpha), \\
k(\alpha+b) & =\gamma(b \alpha) b^{1-2 b \alpha} k(\alpha)
\end{aligned}
$$

и

$$
k(\alpha+c)=\gamma(c \alpha) c^{1-2 c \alpha} k(\alpha)
$$

где

$$
q=b+c, \quad c=b^{-1} \quad \text { и } \quad \gamma(x)=\frac{\Gamma(x)}{\Gamma(1-x)} .
$$

Таким образом, эта функция явно содержит симметрию $\alpha \leftrightarrow q-\alpha$ и является дважды периодической с точностью до некоторых простых множителей. Это целая функция, имеющая только простые нули в точках

$$
(m+1) b+(n+1) c \quad \text { и } \quad-(m b+n c), \quad m, n=0,1,2 \ldots
$$

Следовательно, имеются двойные ряды нулей. Тогда для полной функции $C_{3}\left(\alpha_{a},-\xi / b\right)$ мы имеем

$$
\begin{aligned}
C_{3}\left(\alpha_{a},-\xi / b\right) \rightarrow & \frac{\left(\mu_{r}\right)^{m b}}{(\xi+m b)} \frac{k^{\prime}(0)}{k^{\prime}(-m b)} \times \\
& \times\left[\frac{k\left(2 \alpha_{1}\right) k\left(2 \alpha_{2}\right) k\left(2 \alpha_{3}\right)}{k\left(\alpha_{1}+\alpha_{2}-\alpha_{3}\right) k\left(\alpha_{2}+\alpha_{3}-\alpha_{1}\right) k\left(\alpha_{3}+\alpha_{1}-\alpha_{2}\right)}\right]
\end{aligned}
$$

при $\xi \rightarrow-m b$, и возникает следуюший вопрос: какова правильная экстраполяция $C_{3}\left(\alpha_{a},-\xi / b\right)$, удовлетворяющая этому уравнению?

\section{5. ПРЕДПОЛОЖЕНИЕ ДОЗЗ}

Недавно Дорном и Отто (ДО) [4] и А. и Ал. Замолодчиковыми (З3) [5] было высказано предположение, что простая экстраполящия

$$
C_{3}\left(\alpha_{a},-\xi / b\right)=\left(\mu_{r}\right)^{\xi} \frac{k^{\prime}(0)}{k(-\xi)}\left[\frac{k\left(2 \alpha_{1}\right) k\left(2 \alpha_{2}\right) k\left(2 \alpha_{3}\right)}{k\left(\alpha_{1}+\alpha_{2}-\alpha_{3}\right) k\left(\alpha_{2}+\alpha_{3}-\alpha_{1}\right) k\left(\alpha_{3}+\alpha_{1}-\alpha_{2}\right)}\right]
$$

является правильной. Помимо простоты есть по менњшей мере три сильных аргумента в пользу этого предположения.

1. Максимальная аналитичность. Функция $1 / k(-\xi)$ имеет простые полюсы в точках, заданных (4.7). Замена $1 / k^{\prime}(-\xi)$ на $1 / k(-\xi)$ не приводит к новым полюсам, однако замена на любую другую функцию привела бы к ним. Таким образом, предположение ДОЗЗ минимизирует число вносимых новых полюсов. 
2. Инвариантность по отношению к отражению. Числитель в (5.1) ковариантен по отношению к симметрии отражения в том смысле, что при отражении $\alpha_{a} \rightarrow q-\alpha_{a}$ имеем

$$
k\left(\alpha_{1}\right) k\left(\alpha_{2}\right) k\left(\alpha_{3}\right) \rightarrow R\left(\alpha_{a}\right)\left(k\left(\alpha_{1}\right) k\left(\alpha_{2}\right) k\left(\alpha_{3}\right)\right)
$$

где

$$
R\left(\alpha_{a}\right)=\frac{k\left(2 \alpha_{a}\right)}{k\left(2 \alpha_{a}-q\right)}
$$

при любом выборе $a=1,2,3$. Однако в знаменателе (5.1) в квадратных скобках два множителя меняются местами, а третий заменяется на $k(-\xi)$. Следовательно, в предположении ДОЗЗ числитель (5.1) инвариантен по отношению к отражению, однако другие свойства, связанные с отражением, отсутствуют.

3. Унитарность. Если предположить, что $e^{\alpha \phi(x)}$ образуют полный набор состояний $|\alpha\rangle$, то можно выразить четырехточечные функции в терминах трехточечных функций, вставляя полный набор состояний в соответствии с равенством

$$
\prod_{a=1}^{4}\left\langle\left|e^{\alpha_{a} \phi\left(x_{a}\right)}\right|\right\rangle=\int d \alpha \prod_{i=1}^{2}\langle| e^{\alpha_{i} \phi\left(x_{i}\right)}(|\alpha\rangle\langle\alpha|) \prod_{j=1}^{2}\left\langle e^{\alpha_{j} \phi\left(x_{j}\right)} \mid\right\rangle .
$$

Хотя четырехточечные функции неизвестны для большей части значений параметров $\alpha$, есть некоторые специальные значения, для которых они известны, и Тешнер [11] показал, что для этих значений соотношение (5.4) выполняется, только если $C_{3}$ удовлетворяет предположению ДОЗЗ.

\section{6. ДУАЛЬНОСТЬ}

Если принять предположение ДОЗЗ, то окажется, что коэффициент трехточечной функции обладает только что упоминавшимся свойством отражения, а именно:

$$
G_{N}\left(x_{1}, x_{a}, q-\alpha_{1}, \alpha_{a}\right)=R\left(\alpha_{1}\right) G_{N}\left(x_{1}, x_{a}, \alpha_{1}, \alpha_{a}\right),
$$

и аналогично для $\alpha_{2,3}$. Это показывает, что симметрия $\alpha \rightarrow q-\alpha$, обнаруженная ранее для центра алгебры Вирасоро и примарных полей, распространяется на трехточечные функции, а потому в силу утверждения Тешнера и на $N$-точечные функции.

Наиболее важный результат, однако, состоит в том, что, хотя мы начинали с вычетов в полюсах $\xi=-m b$ интеграла нулевой моды, флуктуирующая часть интеграла приводит к дуальному набору полюсов, а именно к полюсам в точках $\xi=m b+n c$. Инвариантность этой решетки полюсов по отношению к сдвигу $\xi \rightarrow q-\xi$ расширяет дуальность, найденную ранее для центра алгебры Вирасоро и примарных полей, на $N$-точечные функции и, следовательно, на всю теорию. Это окончательное выражение квантовой дуальности, упомянутой в названии статьи.

Наибольшая проблема состоит в том, что свойство дуальности не выполняется для части интеграла по путям, связанной с нулевой модой, поскольку множитель $\Gamma(-\xi / b)$ имеет полюсы только в точках $\xi=m b$. Это означает, что часть интеграла по путям, отвечающая нулевой моде, не согласуется с его остальной частью. Предположение ДОЗЗ 
устраняет это противоречие, но скорее специально для данного случая, посредством замены Г-функции на функцию с двойным набором полюсов. Говоря более точно, функция

$$
\Gamma(-\xi / b) \Gamma(\xi / b+1) \equiv \frac{1}{\sin (\xi / b)} \quad \text { с полюсами в } \quad \xi=m b, \quad m \in Z,
$$

заменяется на функцию

$$
k^{-1}(-\xi) \quad \text { с полюсами в } \quad \xi=m b \quad \text { и } \quad \xi=n c, \quad m, n \in Z,
$$

по модулю $m=0, n \in Z_{+}$, и $n=0, m \in Z_{+}$. Это не вполне удовлетворительно, поскольку произвольно, и в разделе 8 мы предложим интеграл по траекториям, для которого предположение выполняется автоматически, и тем самым противоречие снимаетcя.

\section{7. ДВУХТОЧЕЧНАЯ ФУНКЦИЯ}

Так как два поля $e^{\alpha \phi(x)}$ и $e^{(q-\alpha) \phi(x)}$ имеют одинаковый конформный вес $\Delta_{\alpha}=$ $\alpha(q-\alpha)$, то можно предположить, что каждому конформному весу отвечают также два состояния, что является нежелательным свойством теории. Мы покажем, что это не так, вычисляя двухточечную функцию и интерпретируя ее обычным образом - как произведение двух состояний. Как указывалось выше, из-за $S L(2, C)$-инвариантности интеграла двухточечная функция не может быть вычислена непосредственно, однако она может быть получена как предел

$$
\left\langle e^{\alpha_{1} \phi\left(x_{1}\right)} e^{\alpha_{2} \phi\left(x_{2}\right)}\right\rangle=\lim _{\alpha_{3}=i 0}\left\langle e^{\alpha_{1} \phi\left(x_{1}\right)} e^{\alpha_{2} \phi\left(x_{2}\right)} e^{\alpha_{3} \phi\left(x_{3}\right)}\right\rangle
$$

трехточечной функции, и в этом случае

$$
\begin{aligned}
G_{2}\left(x_{1}, x_{2}, \alpha_{1}, \alpha_{2}\right) & =\left|x_{1}-x_{2}\right|^{2\left(\Delta_{1}+\Delta_{2}\right)}\left(\frac{k(\xi)}{k(-\xi)} \xi+\eta\right) \delta\left(\Delta_{1}-\Delta_{2}\right), \\
\xi & =q-\alpha_{1}-\alpha_{2}, \quad \eta=\alpha_{1}-\alpha_{2} .
\end{aligned}
$$

Последнее выражение имеет все правильные свойства двухточечной функции, и, интерпретируя его как произведение состояний с весом $|x, \alpha\rangle$, находим

$$
\left(\begin{array}{cc}
\langle x, \alpha \mid y, \alpha\rangle & \langle x, \alpha \mid y, q-\alpha\rangle \\
\langle x, q-\alpha \mid y, \alpha\rangle & \langle x, q-\alpha \mid y, q-\alpha\rangle
\end{array}\right)=\left(\begin{array}{cc}
1 & R(\alpha) \\
R^{-1}(\alpha) & 1
\end{array}\right)|x-y|^{4 \Delta},
$$

где $\Delta=\Delta_{1}=\Delta_{2}$, а $R(\alpha)$ - коэффициент отражения, определенный в разделе 5 . Матрица в (7.3) диагонализуема и имеет нулевое собственное значение. Соответственно существует состояние с нулевой нормой, которое отщепляется, а именно $|x, \alpha\rangle-R^{-1}(\alpha)|x, q-\alpha\rangle$. Таким образом, для каждого конформного веса эффективно сушествует только одно состояние. На первый взгляд это выглядит неожиданным, однако на самом деле, как следует из (6.1), это лишь проявление на уровне двухточечных функций инвариантности относительно отражений всех $N$-точечных функций:

$$
\left\langle x, \alpha \mid x_{a}, \alpha_{a} \chi\right\rangle=R(\alpha)\left\langle x, q-\alpha \mid x_{a}, \alpha_{a} \chi\right\rangle, \quad a=2, \ldots, N .
$$

5 Теоретическая и математическая физика, т. 123, № 2, 2000 г. 


\section{8. ОБШЕЕ ПРЕДЛОЖЕНИЕ}

Трудности, связанные с нулевой модой, могут быть преодолены за счет включения рецепта двойного полюса в теорию с самого начала [12] следуюшим образом. Вместо того чтобы определять теорию Лиувилля как теорию одного скалярного поля с экспоненциальным потенциалом, можно определить ее как конформно-инвариантную теорию одного скалярного поля без всяких априорных предположений о виде потенциала. В классической теории потенциал должен иметь конформньй вес $(1,1)$, что приводит к требованию экспоненциальности потенциала. Однако в квантовой теории, благодаря существованию двух примарных полей для каждого конформного веса, потенциал может быть суммой этих двух примарных полей с соответствуюшими коэффициентами, т.е. допускается следуюшая форма интеграла по путям:

$$
\int d \tilde{\phi} \exp \left\{i \int d^{2} x\left(\frac{1}{2}(\partial \tilde{\phi})^{2}+\mu_{b} e^{2 b \tilde{\phi}(x)}+\mu_{c} e^{2 c \tilde{\phi}(x)}+q R \tilde{\phi}\right)\right\} .
$$

Можно убедиться, что необходимым и достаточным условием для вейлевской инвариантности является условие

$$
q=b+c, \quad b c=1 .
$$

Поэтому теория с интегралом по путям (8.1) несомненно является конформно-инвариантной теорией одного скалярного поля. Несколько лет назад такой потенциал уже был предложен в [13]. С этим потенциалом все, что касается структуры двойных полюсов, становится на свои места. В частности:

1. Флуктуируюшая часть интеграла по путям приводит к точно такому же результату, что и в теории с одной экспонентой (для $b c=1$ ). Этот факт замечателен, поскольку форма интеграла гораздо более сложная.

2. Вкладнулевой моды в интегралах по путям обладает нужной структурой двойных полюсов. Точнее говоря, он имеет вид

$$
\frac{1}{\sin (\pi \xi / b)}=b \int d u \frac{\delta(\xi-b u)}{\sin (\pi u)} \longrightarrow \int d u d v \frac{\delta(\xi-b u-c v)}{\sin (\pi u) \sin (\pi v)}=l(\xi),
$$

где $l(\xi)$ - главная часть дилогарифмической функции Барнеса. Функция $l(\xi)$ естественно соответствует функции $k^{\prime}(-\xi) / k(-\xi)$ в том смысле, что имеет простые полюсы в тех же точках, с теми же вычетами: 1 для положительных $\xi$ и -1 для отрицательных $\xi$. Иными словами, она является четной по отношению к симметрии $\alpha \rightarrow q-\alpha$, в то время как функция $k^{\prime}(\xi) / k(\xi)$ является нечетной.

3. Предположение ДОЗЗ автоматически удовлетворяется. Это происходит следующим образом. Из сказанного вьше ясно, что коэффициент трехточечной функции во всех случаях можно записать в виде

$$
h(\xi)\left[\frac{k\left(2 \alpha_{1}\right) k\left(2 \alpha_{2}\right) k\left(2 \alpha_{2}\right)}{k(-\xi) k\left(\alpha_{1}+\alpha_{2}-\alpha_{3}\right) k\left(\alpha_{1}+\alpha_{3}-\alpha_{2}\right) k\left(\alpha_{2}+\alpha_{3}-\alpha_{1}\right)}\right],
$$

где выражение в скобках в точности совпадает с используемым в предположении ДОЗЗ, a $h(\xi)$ есть функция с единичным вычетом, $h(m b+n c)=1$, экстраполяцию которой на 
другие точки следует определить. Утверждение состоит в том, что из трансляционной инвариантности меры следует правило сумм вида

$$
\int d^{2} x_{N}\left(b \mu_{b} \lambda^{b} G_{N+1}\left(x_{I}, x_{N}, \alpha_{I}, b\right)+c \mu_{c} \lambda^{c} G_{N+1}\left(x_{I}, x_{N}, \alpha_{I}, c\right)\right)=\xi G_{N}\left(x_{I}, \alpha_{I}\right)
$$

связываюшее $N$ - и $(N+1)$-точечные функции. Применение этого соотношения к 2 - и 3 -точечным функциям, а также использование условия Вейля и уравнения ренормгруппы приводят к выводу о периодичности функции $h(\xi)$ по отношению к $b$ и $c$. Поскольку при несовместимых действительных $b$ и $c$ единственной такой функцией является константа, то функция $h(\xi)$ есть константа, а ее значение в полюсах равно единице. В случае с одной экспонентой аналогичное рассуж дение приводит лишь к периодичности $h(\xi)$ по $b$.

\section{9. ВЫВОДЫ}

В рамках формализма интеграла по путям описано проявление дуальной симметрии в квантовой теории Лиувилля (в классической теории такая симметрия отсутствует). Дуальность связана с тем, что примарные поля $e^{\alpha \phi(x)}$ и $e^{(q-\alpha) \phi(x)}$ имеют один и тот же конформный вес $\Delta_{\alpha}=\alpha(q-\alpha)$, и проявляется на уровне алгебры Вирасоро и $N$-точечных функций. Дуальность может быть естественно введена в теорию с самого начала за счет использования в интеграле по путям потенциала вида $\mu_{b} e^{b \phi(x)}+\mu_{c} e^{c \phi(x)}$, где $b c=1$.

\section{Список литературы}

[1] H. Poincaré. J. Math. Pure Appl. 1898. V. 5. № 4. P. 157.

[2] A. Polyakov. Phys. Lett. B. 1981. V. 103. P. 207.

[3] J. -L. Gervais, A. Neveu. Nucl. Phys. B. 1984. V. 238. P. 125

[4] H. Dorn, H. -J. Otto. Phys. Lett. B. 1992. V. 291. P. 39; Nucl. Phys. B. 1994. V. 429. P. 375.

[5] A. Zamolodchikov, Al. Zamolodchikov. Nucl. Phys. B. 1996. V. 477. P. 577; Low Dimensional Applications of Quantum Field Theory. Ed. L. Baulieu, V. Kazakov, M. Picco, P. Windey. NATO ASI Series B, Physics Volume 362. Plenum Press, 1997; J.-L. Gervais, A. Neveu. Nucl. Phys. B. 1984. V. 238. P. 396; 1985. V. 257[FS14]. P. 59.

[6] T. Curtright, C. Thorn. Phys. Rev. Lett. 1982. V. 48. P. 1309.

[7] E. Braaten, T. Curtright, C. Thorn. Phys. Lett. 1982. V. 118. P. 115; Ann. Phys. 1983. V. 147. P. 365.

[8] E. D'Hoker, R. Jackiw. Phys. Rev. D. 1982. V. 26. P. 3517.

[9] M. Goulian, M. Li. Phys. Rev. Lett. 1991. V. 66. P. 2051.

[10] Vl. Dotsenko, V. Fateev. Nucl. Phys. B. 1985. V. 251. P. 691.

[11] J. Teschner. Phys. Lett. B. 1995. V. 363. P. 65.

[12] L. O'Raifeartaigh, J. M. Pawlowski, V. V. Sreedhar. Duality in Quantum Liouville Theory. hep-th/9811090.

[13] S. Dotsenko. Mod. Phys. Lett. A. 1991. V. 6. P. 3601. 